\title{
Impact of Homocysteine Level on Long-term Cardiovascular Outcomes in Patients after Coronary Artery Stenting
}

\author{
Jih-Kai Yeh, Chun-Chi Chen, Ming-Jer Hsieh, Ming-Lung Tsai, Chia-Hung Yang, Dong-Yi Chen, \\ Shang-Hung Chang, Chao-Yung Wang, Cheng-Hung Lee and I-Chang Hsieh
}

Department of Cardiology, Percutaneous Coronary Intervention Center, Chang Gung Memorial Hospital, Linkou, Taoyuan, Taiwan

\begin{abstract}
Aim: The prognostic value of homocysteine (HCY) in patients with coronary artery diseases (CAD) is still controversial. The objective of this study was to investigate whether elevated HCY level at admission predict long-term outcomes in patients after percutaneous coronary interventions (PCI) with coronary artery stenting.

Methods: From the institutional registry of Cardiovascular Atherosclerosis and Percutaneous TrAnsluminal INterventions (CAPTAIN), we enrolled a total of 1,307 patients with documented CAD undergone PCI with bare metal stents from July 2003 to December 2014. They were divided into two groups according to the fasting plasma HCY levels before catheterization: group I (883 patients, $<12 \mu \mathrm{mol} / \mathrm{L})$ and group II ( 424 patients, $\geq 12 \mu \mathrm{mol} / \mathrm{L})$. The primary endpoint was occurrence of major adverse cardiac events (MACE), including cardiac death, nonfatal myocardial infarction, stroke, target lesion revascularization, new lesion stenting, and requiring bypass surgery.

Results: After a mean follow-up period of $58 \pm 41$ months, the group II patients had a higher MACE rate $(33.3 \%$ vs. $25.6 \%, p=0.005)$. The main differences between two groups were cardiac death $(8.0 \%$ vs. $3.4 \%, p=0.001)$ and new lesion stenting $(13.6 \%$ vs. $9.5 \%, p=0.034)$. The risks of longterm MACE remained significantly higher in patients with elevated HCY level ( $\geq 12 \mu \mathrm{mol} / \mathrm{L})$ after adjusting for clinical variables, with a hazard ratio of 1.29 (95\% CI, 1.02-1.64, $p=0.036$ ).

Conclusions: Elevated HCY level ( $\geq 12 \mu \mathrm{mol} / \mathrm{L}$ ) was independently associated with increased risk of long-term cardiovascular events in patients after coronary artery bare metal stents implantations. Thus, hyperhomocysteinemia may remain a useful prognostic marker for the risk assessment in clinical care of CAD patients.
\end{abstract}

Key words: Homocysteine, Coronary artery stent, Clinical outcome

\section{Introduction}

Coronary artery disease (CAD) is increasingly prevalent worldwide and is a major cause of cardiovascular mortality. Even if percutaneous coronary intervention (PCI) and effective medical therapies have significantly improved clinical outcomes, certain high risk patients still experienced late cardiovascular events $^{1,2}$. Progression of atherosclerosis and late stent failure were the major causes of late cardiovascular events in these CAD patients. A serum marker with

Address for correspondence: I-Chang Hsieh, No. 5, Fu-Hsing Street, Guishan Township, Taoyuan County, Taiwan

E-mail: hsiehic@ms28.hinet.net (ICH)

Received: May 9, 2016

Accepted for publication: September 25, 2016 prognostic value for late events would help physicians to identify these high-risk patients. Several epidemiologic studies have suggested that elevated homocysteine (HCY) levels are associated with increased risks of atherothrombotic diseases such as CAD, stroke, and peripheral artery disease ${ }^{3-8)}$. Furthermore, Morita $\mathrm{H}$ et al. ${ }^{9)}$ and Schnyder G et al. ${ }^{10)}$ reported HCY levels were associated with the late lumen loss after peripheral artery or coronary artery stenting, respectively. However, the prognostic value of HCY levels was not well studied and the conclusions of past studies were inconsistent ${ }^{11,12)}$. The aim of this study is to clarify the predictive value of plasma HCY levels at index admission for long-term outcomes in patients with coronary artery stenting. 


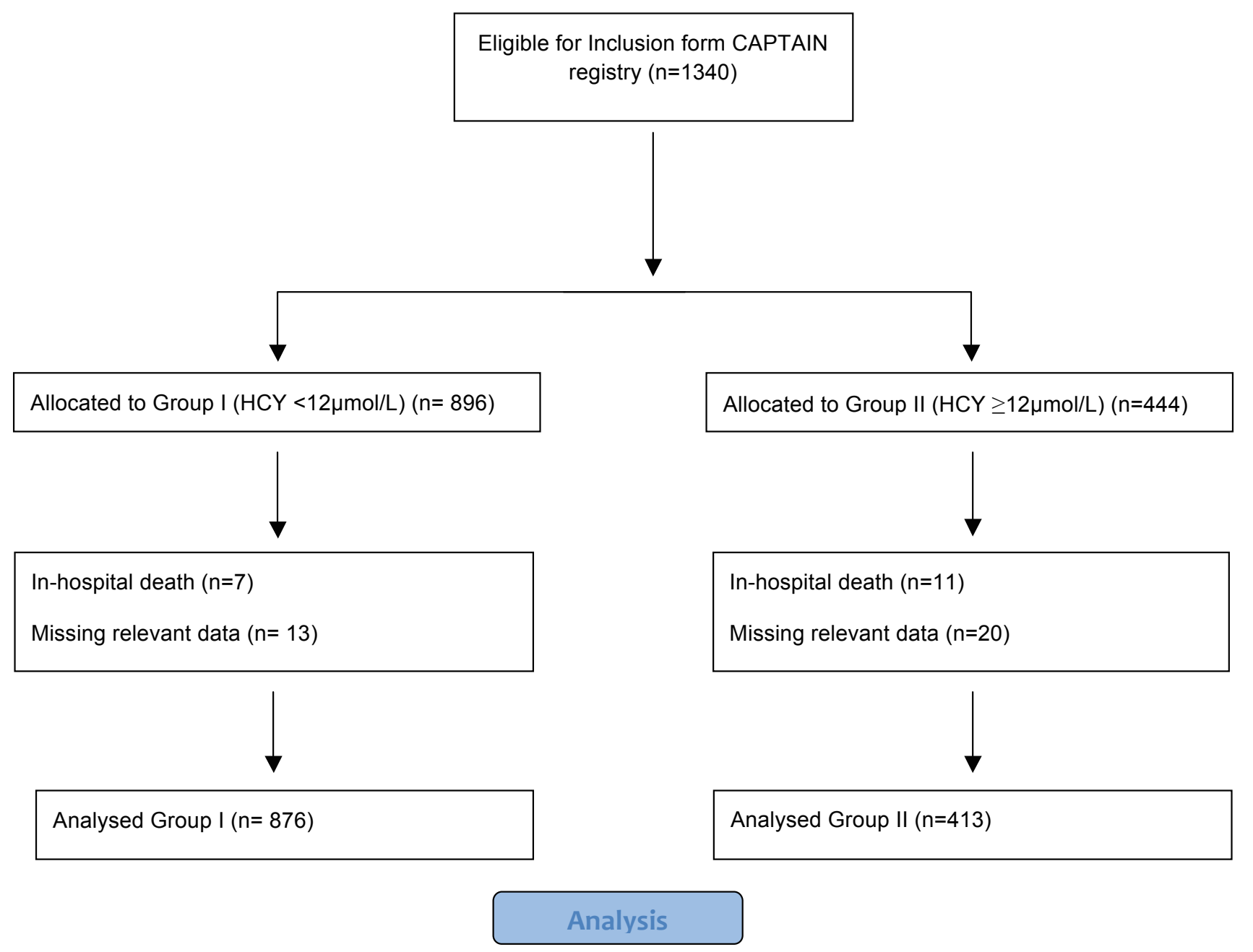

Fig. 1. Flow diagram of patient enrollment and analysis HCY: plasma homocysteine.

\section{Materials and Methods}

\section{Patient Population and Laboratory Analysis}

The Cardiovascular Atherosclerosis and Percutaneous TrAnsluminal INterventions (CAPTAIN) registry, a prospective, physician-initiated, single-center database, which includes 6,300 patients undergoing elective or emergent PCI with stenting at Chang Gung Memorial Hospital. We enrolled consecutive patients in the CAPTAIN registry from July 2003 to December 2014, who had acute myocardial infarction or stable CAD with $>70 \%$ stenosis in coronary arteries underwent stent implantations. To minimize the effects of stent property differences between drug eluting stents and bare metal stents (BMS) on long-term clinical outcomes, only patients with BMS implantations were included into analysis. We excluded patients with severe multivessel disease requiring bypass surgery, intolerable for dual antiplatelet therapy, or not follow the study protocol. Patients with renal insufficiency (serum creatinine level $>1.5 \mathrm{mg} /$ $\mathrm{dL}$ ) or concurrent active infections, inflammatory disorders, or cancer were also excluded to minimize the influence of comorbidities on HCY levels. Stent implantation was performed through the femoral or radial artery according to standard techniques. The type of stent was based on the indication and available stent size. After initial stent deployment, high-pressure balloon inflation $(\geq 14 \mathrm{~atm})$ was applied in most patients. The creatine kinase myocardial-band (CK$\mathrm{MB})$ isoenzyme and troponin I were measured in all of the patients immediately and $6 \mathrm{~h}$ after the procedure to detect periprocedure myocardial infarction (MI). All of the patients received standard ischemia heart disease therapies after procedure, including dual antiplatelet therapy with aspirin and clopidogrel or 
Table 1. Baseline clinical characteristics $(n=1,307)$

\begin{tabular}{|c|c|c|c|}
\hline Homocysteine level & $\begin{array}{c}<12.0 \mu \mathrm{mol} / \mathrm{L} \\
\quad(n=883)\end{array}$ & $\begin{array}{c}\geq 12.0 \mu \mathrm{mol} / \mathrm{L} \\
\quad(n=424)\end{array}$ & $P$ value \\
\hline Age $\pm S D$ (years) & $61.1 \pm 12$ & $65.2 \pm 12$ & $<0.001$ \\
\hline Woman, n (\%) & $169(19.1)$ & $73(17.2)$ & 0.447 \\
\hline Diabetes mellitus, n (\%) & $246(27.9)$ & $153(36.1)$ & 0.003 \\
\hline Hypertension, n (\%) & $497(56.3)$ & $270(63.7)$ & 0.033 \\
\hline Currently smoking, n (\%) & $397(45.0)$ & $170(40.1)$ & 0.107 \\
\hline Hyperlipidemia, n (\%) & $462(51.6)$ & $203(46.1)$ & 0.063 \\
\hline Hx of PCI, n (\%) & $99(11.2)$ & $60(14.2)$ & 0.148 \\
\hline Hx of stroke, n (\%) & $42(4.8)$ & $33(7.8)$ & 0.031 \\
\hline Hx of MI, n (\%) & $468(53.0)$ & $231(54.5)$ & 0.636 \\
\hline Homocysteine, mean \pm SD, $\mu \mathrm{mol} / 1$ & $8.7 \pm 2.0$ & $16.4 \pm 8.5$ & $<0.001$ \\
\hline Serum creatinine, mean $\pm \mathrm{SD}, \mathrm{mg} / \mathrm{dL}$ & $1.01 \pm 0.23$ & $1.12 \pm 0.24$ & $<0.001$ \\
\hline $\mathrm{eGFR}$, mean $\pm \mathrm{SD}, \mathrm{ml} / \mathrm{min} / 1.73 \mathrm{~m}^{2}$ & $74.9 \pm 26.8$ & $65.8 \pm 20.1$ & $<0.001$ \\
\hline Stable angina, $\mathrm{n}(\%)$ & $486(55.0)$ & $242(57.1)$ & 0.488 \\
\hline ACS, n (\%) & $397(45.0)$ & $182(42.9)$ & 0.521 \\
\hline LVEF < 40\%, n (\%) & $76(9.3)$ & $70(17.9)$ & $<0.001$ \\
\hline Cardiogenic shock, n (\%) & $46(5.2)$ & $27(6.4)$ & 0.440 \\
\hline Multivessel disease, $\mathrm{n}(\%)$ & $578(65.5)$ & $295(69.6)$ & 0.149 \\
\hline
\end{tabular}

ACS: acute coronary syndrome; eGFR: estimated glomerular filtration rate, calculated by the Modification of Diet in Renal Disease (MDRD) equation. Hx: history of occurrence 6 months or longer before the study; LVEF: left ventricular ejection fraction, measured by angiography; MI: myocardial infarction; PCI: percutaneous coronary intervention; SD: standard deviation.

ticagrelor, beta-blockers, statins, angiotensin converting enzyme inhibitors or angiotensin receptor blockers. Blood samples for plasma total HCY were collected after overnight fasting before index coronary angiography and intervention. The plasma total HCY level was measured by high-performance liquid chromatography with fluorescence detection (Hitachi Labospect 008, AS, Japan). We use a cutoff value of $12 \mu \mathrm{mol} / \mathrm{L}$ for hyperhomocysteinemia ${ }^{10)}$. The study protocol was approved by the Institutional Review Board of Chang Gung Medical Foundation. All participants provided informed consents to undergo the procedure and follow-up protocol.

\section{Angiographic Evaluation}

The minimal luminal diameter (MLD), reference vessel diameter (RVD), percent of diameter stenosis (DS\%), and balloon diameter were measured from multiple projections using the automatic edge-detection method. Acute gain was defined as the difference in MLD between before and after stenting. The quantitative coronary angiographic analysis was performed by two experienced interventional cardiologists, and images were selected with a end-diastolic cine-frame of the most severe and nonforeshortened projection. A contrast-filled guiding catheter was used as a reference for calibration. The interobserver correlation coefficient $(r)$ was $0.93(p<0.01)$, and the intraobserver correlation coefficient was $0.95(p<0.01)$.

\section{Definitions of the Endpoints}

The study endpoint was the occurrence of major adverse cardiac events (MACE), a composite of cardiac death, nonfatal MI, stroke, target lesion revascularization, new lesion stenting, and necessitation of coronary bypass surgery. Cardiac death was defined as any death due to immediate cardiac cause, such as MI, low-output pumping failure, or fatal arrhythmia. The MI was diagnosed if the patient experienced prolonged chest pain, ischemic ST-T changes shown on electrocardiography, and elevated CK-MB isoenzyme or troponin I levels. Clinical follow-up visits at the outpatient clinics were scheduled at 1, 2, 3, 6, 9, and 12 months, and every 3 months thereafter. Information regarding clinical events was obtained from hospital medical records, the referring physician, and phone interviews with the patients or their relatives through December 30, 2015.

\section{Statistical Analysis}

Data were prospectively collected and analyzed using SPSS statistical software package (version 22.0, IBM) for all statistical analyses. Continuous variables were represented as means \pm standard deviation (SD) and compared between groups using a two-tailed $t$ test. Categorical variables were presented as numbers 
Table 2. Angiographic and PCI-related characteristics $(n=1,604)$

\begin{tabular}{|c|c|c|c|}
\hline Homocysteine level & $\begin{array}{c}<12.0 \mu \mathrm{mol} / \mathrm{L} \\
\quad(n=1082)\end{array}$ & $\begin{array}{c}\geq 12.0 \mu \mathrm{mol} / \mathrm{L} \\
\quad(n=522)\end{array}$ & $P$ value \\
\hline Complex lesion (type $\mathrm{B} 2$ or $\left.\mathrm{C}^{*}\right)$, n (\%) & $832(76.9)$ & $421(80.7)$ & 0.094 \\
\hline Target vessel site & & & 0.924 \\
\hline Left main, $\mathrm{n}(\%)$ & $24(2.2)$ & $14(2.7)$ & \\
\hline $\mathrm{LAD}, \mathrm{n}(\%)$ & $442(40.9)$ & $221(42.3)$ & \\
\hline LCx, n (\%) & $207(19.1)$ & $94(18.0)$ & \\
\hline RCA, n (\%) & $402(37.2)$ & $189(36.2)$ & \\
\hline Graft, n (\%) & $7(0.6)$ & $4(0.8)$ & \\
\hline Ostial lesion, n (\%) & $78(7.2)$ & $39(7.5)$ & 0.838 \\
\hline Bifurcation, n (\%) & $76(7.0)$ & $31(5.9)$ & 0.456 \\
\hline Lesion length, mean $\pm \mathrm{SD}(\mathrm{mm})$ & $20.2 \pm 10$ & $20.5 \pm 11$ & 0.629 \\
\hline Stent size, mean $\pm \mathrm{SD}(\mathrm{mm})$ & $3.3 \pm 0.7$ & $3.3 \pm 0.6$ & 0.470 \\
\hline Calcified, n (\%) & $126(11.6)$ & $89(17.0)$ & 0.004 \\
\hline Thrombus containing, $\mathrm{n}(\%)$ & $163(15.1)$ & $58(11.1)$ & 0.037 \\
\hline Number of stents per lesion & 1.10 & 1.12 & 0.178 \\
\hline \multicolumn{4}{|l|}{ Pre-procedure target lesion } \\
\hline$\%$ diameter stenosis, mean \pm SD & $82.5 \pm 13$ & $82.9 \pm 13$ & 0.547 \\
\hline $\operatorname{MLD}(\mathrm{mm})$, mean $\pm \mathrm{SD}$ & $0.58 \pm 0.5$ & $0.57 \pm 0.5$ & 0.627 \\
\hline $\operatorname{RVD}(\mathrm{mm})$, mean $\pm \mathrm{SD}$ & $3.32 \pm 0.6$ & $3.33 \pm 0.6$ & 0.884 \\
\hline \multicolumn{4}{|l|}{ Post-procedure target lesion } \\
\hline$\%$ diameter stenosis, mean $\pm \mathrm{SD}$ & $6.4 \pm 6$ & $6.6 \pm 6$ & 0.523 \\
\hline $\operatorname{MLD}(\mathrm{mm})$, mean $\pm \mathrm{SD}$ & $3.36 \pm 0.5$ & $3.10 \pm 0.5$ & 0.479 \\
\hline $\operatorname{RVD}(\mathrm{mm})$, mean $\pm S D$ & $3.32 \pm 0.6$ & $3.36 \pm 0.6$ & 0.894 \\
\hline Acute gain $(\mathrm{mm})$, mean $\pm S D$ & $2.52 \pm 0.6$ & $2.53 \pm 0.6$ & 0.812 \\
\hline
\end{tabular}

LAD: left anterior descending artery; LCx: left circumflex artery; LIMA: left internal mammary artery; OM: obtuse margin; MLD: minimal luminal diameter; PCI percutaneous coronary intervention; PDA: posterior descending artery; PL: posterolateral artery; RCA: right coronary artery; RVD: reference vessel diameter; SD: standard deviation.

* A complex lesion was defined as a lesion of type B2 and C according to the classification of the American College of Cardiology and the American Heart Association.

(percentages) and compared with a Fisher's exact test. Cox proportional hazard regression models were used to determine the relationship between plasma HCY levels and MACE rates. They were presented as hazard ratios (HRs) with 95\% confidence intervals (CIs) and adjusted for confounding factors including variants in clinical and angiographic characteristics. MACE-free survival curve was depicted by the Kaplan-Meier method and differences between the two groups were assessed by the log-rank test. A $p$ value less than 0.05 was considered statistically significant.

\section{Results}

\section{Baseline Characteristics}

A total of 1,340 patients were in accordance with eligibility criteria for study participants in the CAPTAIN registry. There were 33 patients excluded from relevant analyses for missing data. Finally, 1,307 patients (mean age 62 \pm 12 years, range $24-93$ years;
81.5\% male) with 1,604 lesions and 1,800 BMS implantations were consecutively enrolled for analyses (Fig. 1). The patients were divided into two groups according to baseline plasma total HCY level with a cutoff value of $12 \mu \mathrm{mol} / \mathrm{L}$ : group I (883 patients, $\mathrm{HCY}<12 \mu \mathrm{mol} / \mathrm{L})$, group II (424 patients, $\geq 12$ $\mu \mathrm{mol} / \mathrm{L})$. Comparisons of the baseline characteristics are shown in Table 1. The patients in group II were significantly older $(65.2 \pm 12$ vs. $61.1 \pm 12$ years, $p<$ $0.001)$, and there were more patients with diabetes (36.1\% vs. $27.9 \%, p=0.003)$, hypertension $(63.7 \%$ vs. $56.3 \%, p=0.033)$, history of stroke $(7.8 \%$ vs. $4.8 \%$, $p=0.031)$, and left ventricular systolic dysfunction (ejection fraction $<40 \%$ ) at the initial event $(17.9 \%$ vs. $9.3 \%, p<0.001)$. The mean plasma total $\mathrm{HCY}$ levels in group I and II were $8.7 \mu \mathrm{mol} / \mathrm{L}$ and 16.4 $\mu \mathrm{mol} / \mathrm{L}$, respectively (Table $\mathbf{1}$ ). The mean serum creatinine level in group II was higher than that in group I $(1.12 \pm 0.24$ vs. $1.01 \pm 0.24 \mathrm{mg} / \mathrm{dL}, p<0.001)$ and the mean estimated glomerular filtration rates (eGFR), 
Table 3. In-hospital cardiac events

\begin{tabular}{lccc}
\hline \multicolumn{1}{c}{ Homocysteine level } & $<12.0 \mu \mathrm{mol} / \mathrm{L}$ & $\geq 12.0 \mu \mathrm{mol} / \mathrm{L}$ & $P$ value \\
\hline Number of patients & 883 & 424 & 0.004 \\
Death, n (\%) & $6(0.7)$ & $12(2.9)$ & 0.014 \\
$\quad$ Cardiac & $6(0.7)$ & $10(2.4)$ & 0.105 \\
$\quad$ Non-Cardiac & $0(0)$ & $2(0.5)$ & 0.607 \\
Non-fatal MI, n (\%) & & & 1.00 \\
$\quad$ NSTEMI & $2(2.0)$ & $0(1.9)$ & 1.00 \\
$\quad$ STEMI & $3(0.2)$ & $2(0.5)$ & 0.662 \\
Acute stent thrombosis, n (\%) & $5(0.6)$ & $4(0.9)$ & 0.483 \\
Subacute stent thrombosis, n (\%) & $2(0.2)$ & $1(0.2)$ & 1.000 \\
Emergent bypass surgery, n (\%) & $2(0.2)$ & 0 & 1.00 \\
Non-fatal stroke, n (\%) & $33(3.7)$ & $19(4.5)$ & 0.546 \\
MACE, n (\%) & & 0 \\
\hline
\end{tabular}

MACE: major adverse cardiac event; MI: myocardial infarction; NSTEMI: non ST-segment elevation myocardial infarction; STEMI: ST- segment elevation myocardial infarction

Table 4. Clinical events during long-term follow-up ( $n=1,289,58 \pm 41$ months)

\begin{tabular}{lccc}
\hline \multicolumn{1}{c}{ Homocysteine level } & $<12.0 \mu \mathrm{mol} / \mathrm{L}$ & $\geq 12.0 \mu \mathrm{mol} / \mathrm{L}$ & $P$ value \\
\hline Number of patients & 876 & 413 & 0.001 \\
Overall mortality, n (\%) & $56(6.4)$ & $50(12.1)$ & 0.001 \\
$\quad$ Cardiac, n (\%) & $30(3.4)$ & $33(8.0)$ & 0.318 \\
$\quad$ Non-cardiac, n (\%) & $26(3.0)$ & $17(4.1)$ & 0.450 \\
Non-fatal MI, n (\%) & $31(3.5)$ & $18(3.8)$ & 0.494 \\
Target lesion revascularization, n (\%) & $87(9.9)$ & $46(11.2)$ & 0.034 \\
New lesion stenting, n (\%) & $83(9.5)$ & $56(13.6)$ & 0.659 \\
Coronary bypass surgery, n (\%) & $15(1.7)$ & $9(2.2)$ & 1.00 \\
Non-fatal stroke, n (\%) & $19(2.2)$ & $9(2.2)$ & 0.005 \\
MACE, n (\%) & $224(25.6)$ & $137(33.3)$ & \\
\hline
\end{tabular}

MACE: major adverse cardiac events; MI: myocardial infarction

calculated by the Modification of Diet in Renal Disease (MDRD) equation, in group I and II were $74.9 \pm 26.8$ and $65.8 \pm 20.1 \mathrm{~mL} / \mathrm{min} / 1.73 \mathrm{~m}^{2}$, respectively $(p<0.001)$.

\section{Angiographic Characteristics}

There were total 1,082 lesions in group I and 522 lesions in group II, of which 1,253 (78.1\%) were complex lesions (type B2 and C), 107 (6.7\%) were bifurcation lesions, and $117(7.3 \%)$ were ostial lesions. The mean lesion length was $20 \pm 10 \mathrm{~mm}$. There was no significant difference in lesion location, length, or complexity between the two groups. However, patients in groups II had more calcified lesions $(17.0 \%$ vs. $11.6 \%, p=0.004)$ and fewer thrombus-containing lesions (11.1\% vs. $15.1 \%, p=0.037)$. For PCI-related characteristics, there was no significant difference in the pre- or post-procedure DS\%, MLD, or RVD of the target lesions. Also, there was no significant difference in the sizes and lengths of the implanted coronary artery stents between the two groups (Table 2). A total of 1,800 stents were implanted including 40 Bx Velocity (Johnson and Johnson, Miami Lakes, Florida, USA), 427 Driver (Medtronic, Santa Rosa, CA, USA), 122 Express (Boston Scientific, Natick, Massachusetts, USA), 45 Integrity (Medtronic), 88 Liberte (Boston Scientific), 14 Multilink (Guidant, Santa Clara, CA, USA), 1 Multi-Link TETRA (Guidant), 312 Multi-Link PENTA (Guidant), 76 Multi-Link PIXEL (Guidant), 469 Multi-Link VISION (Abbott Vascular, Santa Clara, CA, USA), 53 Multi-Link ZETA (Abbott), 27 Omega (Boston Scientific), 61 R (OrbusNeich, Hong Kong), and 65 S7 (Medtronic) stents.

\section{In-Hospital Cardiac Events}

The in-hospital cardiac events in the two groups 


\section{Kaplan- Meier Survival Estimates}

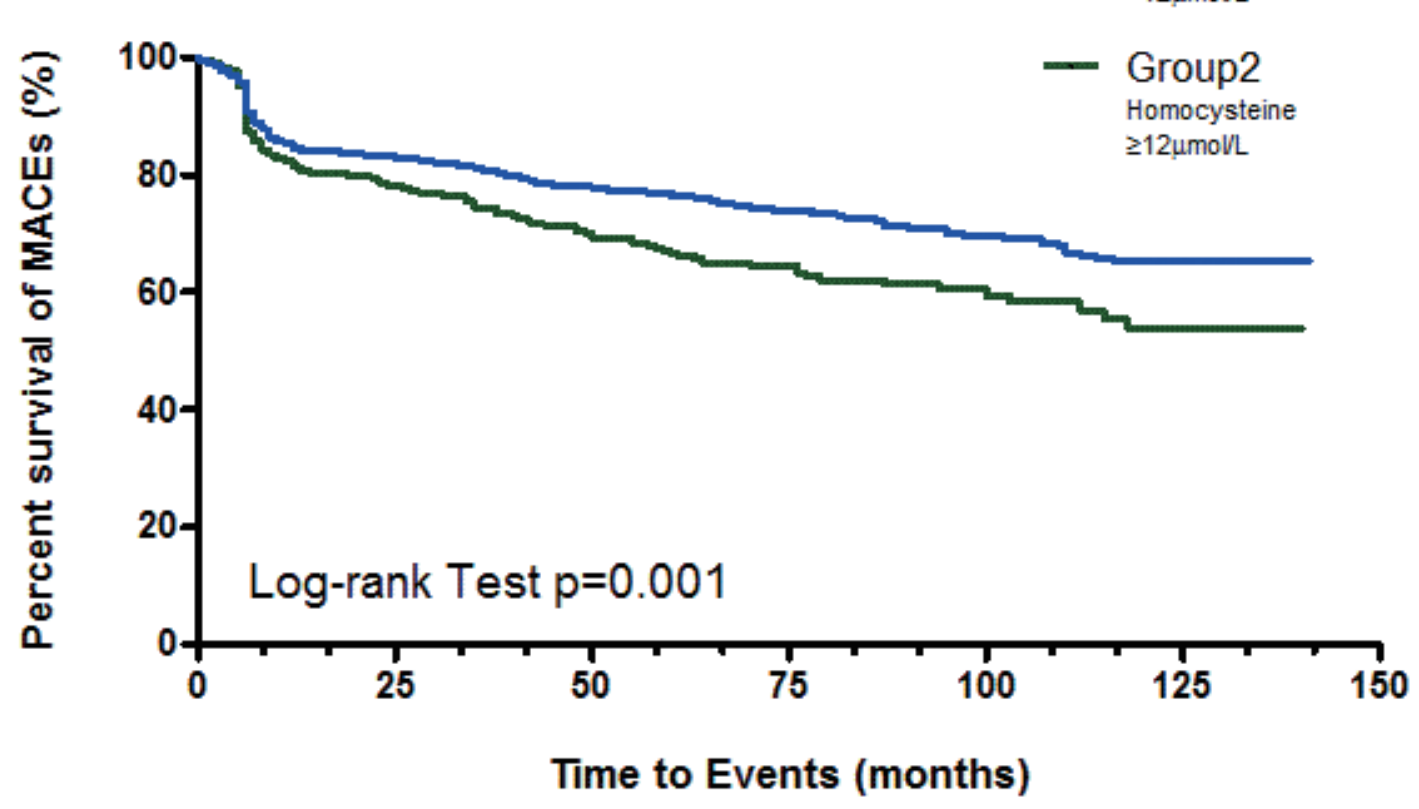

Fig. 2. The MACEs-free survival by Kaplan-Meier analysis between groups

The event-free survival rate was significantly higher in the group I patients with lower homocysteine levels at admission $(<12 \mu \mathrm{mol} / \mathrm{L})(\mathrm{Log}$-rank test, $p=0.001)$.

MACE: major adverse cardiac event.

are shown in Table 3. The overall and cardiac-related mortality rates were higher in group II than in group I $(2.9 \%$ vs. $0.7 \%, p=0.004 ; 2.4 \%$ vs. $0.7 \%, p=0.014$, respectively). However, there were no significant differences in the incidence of MI, acute, and subacute stent thrombosis, emergency bypass surgery, nonfatal stroke, or in-hospital MACE between the two groups.

\section{Long-Term Clinical Outcomes}

A total of 1,289 patients, who survived and discharged from hospital after PCI, were followed up for a mean of $58 \pm 41$ months. The patients in group II had a significantly higher mortality rate than those in group I $(12.1 \%$ vs. $6.4 \%, p=0.001)$ and majority were from cardiac death $(8.0 \%$ vs. $3.4 \%, p=0.001)$. In addition, the patients in group II had a higher percentage of new coronary artery lesions requiring stenting than patients in group I $(13.6 \%$ vs. $9.5 \%, p=$ 0.034). Overall, the group II patients had a higher MACE rate than group I patients $(33.3 \%$ vs. $25.6 \%$, $p=0.005)$ (Table 4). In Kaplan-Meier analysis, there was a significant difference in the MACE-free survival rate between the two groups (log-rank test; $p=0.001$ ) (Fig. 2). The univariate Cox proportional hazards analysis showed that the MACE rates were significantly associated with diabetes mellitus, LVEF $<40 \%$, mul- tivessel disease, complex type $\mathrm{B} 2$ or $\mathrm{C}$ lesion, postprocedure MLD, serum creatinine level, and plasma HCY level $\geq 12 \mu \mathrm{mol} / \mathrm{L}$. After adjusting for factors of age, gender, hypertension, diabetes mellitus, hyperlipidemia, serum creatinine, HCY level $>12 \mu \mathrm{mol} / \mathrm{L}$, multivessel disease, type $\mathrm{B} 2$ or $\mathrm{C}$ lesions, post-procedure MLD, and LVEF $<40 \%$ in multivariable analysis, elevated plasma HCY levels $(\geq 12 \mu \mathrm{mol} / \mathrm{L})$ remained a statistically significant predictor for longterm cardiovascular outcomes, with a HR of 1.29 (95\% CI: $1.02-1.64, p=0.036)$. Other significant predictors were LVEF < 40\% (HR: 1.52, 95\% CI: $1.11-$ 2.09, $p=0.010$ ), multivessel disease (HR: 1.51, 95\% CI: $1.17-1.95, p=0.002$ ), post-procedure MLD (HR: $0.66,95 \%$ CI: $0.53-0.83, p<0.001$ ), and serum creatinine (HR: 1.69, 95\% CI: $1.06-2.71, p=0.027$ ) (Table 5). Moreover, the plasma HCY values were divided into quintiles to examine their relationship with the long-term MACEs (Table 6). There was a graded association between HCY levels and MACE rates, which compared with quintiles $1-3$, the adjusted HRs in quintile 4 (HCY range 11.3-13.4 $\mu \mathrm{mol} / \mathrm{L}$ ) and quintile 5 ( $\mathrm{HCY}$ range $>13.4 \mu \mathrm{mol} / \mathrm{L}$ ) were 1.06 (95\% CI: $0.78-1.44)$ and 1.37 (95\% CI: $1.03-1.82$ ), respectively. 
Table 5. Predictors of long-term major cardiac events

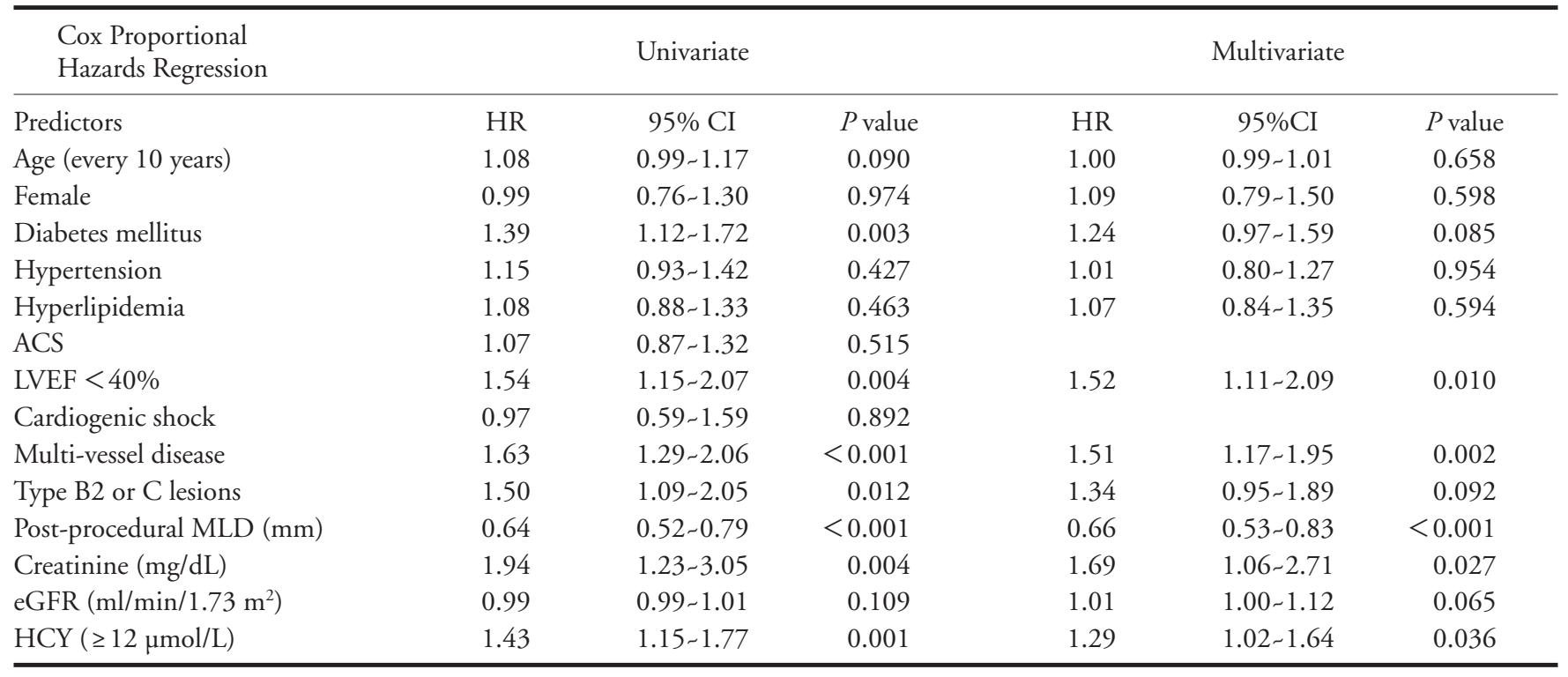

ACS: acute coronary syndrome; eGFR: estimated glomerular filtration rate; LVEF: left ventricular ejection fraction; MLD: minimal luminal diameter; HCY: plasma total homocysteine level.

Table 6. Long-term outcomes by homocysteine quintiles

\begin{tabular}{lccccccc}
\hline & Patient number & HCY range & Event number & Event rate & *Adjusted HR & $95 \%$ CI & $P$ value \\
\hline Quintile 1-3 & 789 & $<11.3$ & 201 & 25.4 & 1 & - \\
Quintile 4 & 293 & $11.3-13.4$ & 84 & 28.7 & 1.06 & $0.78 \sim 1.44$ & 0.697 \\
Quintile 5 & 206 & $>13.4$ & 75 & 36.4 & 1.37 & $1.03 \sim 1.82$ & 0.032 \\
\hline
\end{tabular}

*Adjusted HR: hazard ratio adjusted for age, sex, hypertension, diabetes mellitus, hyperlipidemia, serum creatinine, multi-vessel disease, post procedure MLD, and LVEF $<40 \%$

HCY: plasma total homocysteine level. LVEF: left ventricular ejection fraction; MLD: minimal luminal diameter.

\section{Discussion}

In our retrospective analysis of a single center registry, 1,307 patients with coronary artery diseases after bare metal stent implantation were included for nearly 5 years follow-up. The patients with hyperhomocysteinemia $(\geq 12 \mu \mathrm{mol} / \mathrm{L})$ had a higher long-term MACE rate, mainly from cardiac mortality and new lesion stenting. After adjusting for multiple clinical, angiographic, and PCI-related variables, the association remained significant (HR, 1.29; 95\% CI: $1.02-$ 1.64). There is a dose dependent association between HCY levels and risks for long-term MACE, where the adjusted HRs in quintile 4 (HCY range 11.3-13.4 $\mu \mathrm{mol} / \mathrm{L}$ ) and quintile 5 (HCY range $>13.4 \mu \mathrm{mol} / \mathrm{L}$ ) were 1.06 (95\% CI: $0.78-1.44)$ and 1.37 (95\% CI: $1.03-1.82)$, respectively.

Since McCully et al. ${ }^{13)}$ described vascular diseases in patients with severe hyperhomocysteinemia $(>100$ $\mu \mathrm{mol} / \mathrm{L})$ in 1969 , subsequent case control and epide- miologic studies have shown a strong association between plasma HCY levels and the risks of atherosclerotic diseases. As observational studies have shown, even mildly elevated plasma HCY levels (between 11.4 and $14.3 \mu \mathrm{mol} / \mathrm{L}$ ) increase the risk of cardiovascular events $^{14,15)}$. However, the pathophysiological mechanisms were not fully understood. As implied from basic research on the mechanism of HCY associated with vascular injury ${ }^{16-19)}$, HCY produced reactive oxygen species and caused lipid peroxidation, platelets and leukocytes activation, and increased prothrombotic factors, which resulted in vascular inflammation, thrombus formation. Furthermore, HCY causes endothelial dysfunction with impaired release of nitric oxide and vasodilatation in response to shear stress in vascular wall, which predisposes to vulnerable plagues rupture, contributes to atheroma progression, and ultimately gives rise to vascular events. Case control studies showed HCY level was significantly higher in patients with acute coronary syndrome $(\mathrm{ACS})^{20,21)}$. 
In regard to the role of $\mathrm{HCY}$ in PCI, researchers also reported that patients with coronary slow flow phenomenon had higher HCY levels ${ }^{22)}$, increased oxidative stress markers and impaired endothelial cell function $^{23)}$. Although hyperhomocysteinemia was considered to be associated with more ACS and periprocedural acute thrombotic events in previous reports, there was no significant difference in incidence of initial ACS presentation and in-hospital acute events between two groups in our study. We thought it could be explained by two main reasons. Frist, insufficient numbers of in-hospital thrombotic events and relatively mild to moderate elevated HCY levels in both groups are not enough to detect the difference in the prespecified clinical outcomes. Second, the thrombotic events in CAD caused from a complex interaction between numerous factors from patient-related, pharmacologic-related, procedural-related, or devicerelated related factors that might be unequal distribution between two groups in a nonrandomized trial.

With respect of the issue of increased cardiac mortality in patients with hyperhomcysteinemia, some relevant clinical observation results might explain this background issue. First, HCY levels correlated to the severity and extent of coronary atherosclerosis ${ }^{24)}$ and associated with vascular calcification ${ }^{25,26)}$ and unstable atherosclerotic plagues ${ }^{27)}$. These anatomic factors increased the difficulties of coronary intervention and associated with acute complications and worse longterm clinical outcomes. Second, the prothrombotic and proinflammatory effects of $\mathrm{HCY}$ are associated with coronary slow flow during intervention and may impair angiogenesis and collateral vessels development ${ }^{28)}$, resulting in inadequate myocardial reperfusion, increased extent of myocardial necrosis, and finally ventricular pumping failure and worse longterm outcomes. However, we did not prospectively include the prespecified variables, such as vulnerability of atherosclerotic plagues or quantification of coronary slow flow during PCI in our initial study protocol and thus, there was no complete clinical data to analysis this association. Nevertheless, this issue is sufficiently noteworthy to merit additional well-designed studies.

In human studies, Kosokabe et al. ${ }^{29)}$ observed a positive correlation between plasma HCY levels and atherosclerotic plaque areas in reference segments of coronary arteries, which were measured by intravascular ultrasound 6 months after the intervention. This evidence supports the atherogenic propensity of $\mathrm{HCY}$ and also could explain our study results, which more numbers of new lesions stenting and worse long-term outcomes in our patients with hyperhomocystinemia.

However, the results in past studies were inconsistent. In the late $1990 \mathrm{~s}$, Nygard et al. ${ }^{30)}$ reported a strong and graded relationship between plasma HCY levels and the overall mortality in 587 patients with angiographically confirmed CAD after a median follow-up of 4.6 years. In their study, patients with a homocysteine level $>15 \mathrm{mmol} / \mathrm{L}$ had a $24.7 \%$ total mortality rate, compared with $3.8 \%$ for those with a homocysteine level $<9 \mathrm{mmol} / \mathrm{L} \quad(p=0.02)$. Later, Stubbs et al. ${ }^{31)}$ also stated the similar association of plasma HCY levels and the long-term prognosis (median 2.5 years) in 440 patients with acute coronary syndrome, in which patients with a HCY level $(>12.2 \mu \mathrm{mol} / \mathrm{L})$ had a 2.6 -fold higher risk of cardiac death and myocardial re-infarction. In contrast, a prospective observational study by Zairis et al. ${ }^{12)}$, including 483 patients with either stable angina or acute coronary syndrome after successful coronary stenting, found no significant association between plasma $\mathrm{HCY}$ levels and the composite endpoint of cardiac death, MI, or re-hospitalization for angina after a median follow-up of 22 months. The disparate results may be attributed to the shorter follow-up duration and different enrolled patient population and study endpoints in later study. In Zairis et al. study, patients with CAD and LVEF $<35 \%$, implicating severe and extensive disease may have a higher plasma HCY level and poor prognosis ${ }^{4)}$, were excluded. Patients with ST segment elevation myocardial infarction (STEMI) or non-ST segment elevation myocardial infarction (NSTEMI) presented more than $6 \mathrm{~h}$ or $24 \mathrm{~h}$ of index pain, respectively, were excluded. The endpoint of rehospitalization for angina may not be objectively and difficult to differentiate in some clinical situation. These enrolled criteria and study endpoints were also different from our study.

Even though evidence has shown an association between elevated plasma HCY levels and increased risks of late cardiac events and poor prognosis in patients with $\mathrm{CAD}$, in randomized controlled trials, secondary prevention management with folic acid and vitamins failed to prevent or decrease cardiovascular events, even decreasing homocysteine levels ${ }^{32-34)}$. However, it seems too early to make a conclusion before further welldesigned clinical trials with different dosages or agents were performed. A longer follow-up period and specific patient population with moderately elevated homocysteine levels may be necessary to observe impacts of HCY on progression of coronary atherosclerosis and cause late stent failure and clinical events. In fact, a recent meta-analysis on patients with chronic kidney disease found significant benefits of reducing risks of cardiovascular disease from folate treatment, especially in those with a higher baseline homocysteine level (mean $>25 \mu \mathrm{mol} / \mathrm{L}$, relative risk 0.87, $p=0.049)^{35)}$. The further research to investigate the pathogenesis of 
$\mathrm{HCY}$ in atherosclerotic disease is important to contribute a newly potential therapeutic target to reduce homocysteine levels and improve outcomes.

\section{Limitations}

There were several limitations in this study. First, we used a cutoff value of HCY levels $12 \mu \mathrm{mol} / \mathrm{L}$ make uneventful distribution of the study population. Second, the optimal timing for blood collection and testing methods for plasma HCY levels have not been well defined. We sample blood in early morning after fasting for more than $12 \mathrm{~h}$ and within $24 \mathrm{~h}$ after admission. HCY levels may influence by numerous environmental factors such as dietary contents, which we could not estimate and adjust. Third, the methylene tetrahydrofolate reductase (MTHFR) enzyme, folic acid, vitamin $\mathrm{B} 6$, and vitamin $\mathrm{B} 12$ are required for metabolizing homocysteine to methionine or cysteine. Gene polymorphism of MTHFR C677T and dietary deficiency of folic acid, vitamin B6, or vitamin B12 are associated with hyperhomocysteinemia. In our study protocol, we did not routinely measure serum folate, vitamin $\mathrm{B} 12$, and gene polymorphism. Thus, we could not analyze the interaction between folic acid, vitamin B12, gene polymorphism, and risks of cardiovascular events. However, the association of MTHFR genotype and cardiovascular events is still controversial, and it could be modified by the folate status, where high folate levels reduced the effect of MTHFR C677T on HCY levels ${ }^{7,36}$. Thus, we suggested that HCY levels reflect the influence of the gene-environment interactions and should be a more relevant factor to predict cardiovascular outcomes. Fourth, this is a single center prospective study and the results may not be applicable to other racial and ethnic groups.

\section{Conclusions}

An elevated HCY level $(\geq 12 \mu \mathrm{mol} / \mathrm{L})$ increased the risk of long-term cardiovascular events in patients who undergo intracoronary BMS implantations. After adjusting for clinical, angiographic, and PCI-related variables, an elevated homocysteine level remained a robust independent predictor for long-term clinical outcomes. Even randomized trials failed to demonstrate clinical benefits of HCY reducing therapies, HCY levels remain a useful clinical predictive marker for early identification of high-risk patients with worse long-term outcomes after coronary artery stenting.

\section{Acknowledgments}

Thanks for Victor Chien-Chia Wu contributed towards the study by English editing.

\section{COI}

There is no conflict of interest to declare in relation to this work.

\section{Source of Funding}

This work was supported by the grant from Chang Gung Memorial Hospital [grant number: CORPG 3C0162]

\section{References}

1) Elezi S, Kastrati A, Pache J, Wehinger A, Hadamitzky M, Dirschinger J, Neumann FJ, Schömig A: Diabetes mellitus and the clinical and angiographic outcome after coronary stent placement. J Am Coll Cardiol. 1998; 32: 1866-1873

2) Abizaid A, Kornowski R, Mintz GS, Hong MK, Abizaid AS, Mehran R, Pichard AD, Kent KM, Satler LF, Wu H, Popma JJ, Leon MB: The influence of diabetes mellitus on acute and late clinical outcomes following coronary stent implantation. J Am Coll Cardiol. 1998; 32: 584-589

3) Wald DS, Law M, Morris JK: Homocysteine and cardiovascular disease: evidence on causality from a meta-analysis. BMJ. 2002; 325: 1202

4) Schaffer A, Verdoia M, Cassetti E, Marino P, Suryapranata H, De Luca G; Novara Atherosclerosis Study Group (NAS): Relationship between homocysteine and coronary artery disease. Results from a large prospective cohort study. Thromb Res. 2014; 134: 288-293

5) Homocysteine Studies Collaboration: Homocysteine and risk of ischemic heart disease and stroke. JAMA. 2002; 288: 2015-2022

6) Casas JP, Bautista LE, Smeeth L, Sharma P, Hingorani AD: Homocysteine and stroke: evidence on a causal link from mendelian randomisation. Lancet. 2005; 365: 224-232

7) Klerk M, Verhoef P, Clarke R, Blom HJ, Kok FJ, Schouten EG; MTHFR Studies Collaboration Group. MTHFR $677 \mathrm{C} \rightarrow \mathrm{T}$ polymorphism and risk of coronary heart disease. JAMA. 2002; 288: 2023-2031

8) den Heijer M, Koster T, Blom HJ, Bos GM, Briet E, Reitsma PH, Vandenbroucke JP, Rosendaal FR: Hyperhomocysteinemia as a risk factor for deep-vein thrombosis. N Engl J Med. 1996; 334: 759-762

9) Morita H, Kurihara H, Yoshida S, Saito Y, Shindo T, OhHashi Y, Kurihara Y, Yazaki Y, Nagai R: Diet-induced hyperhomocysteinemia exacerbates neointima formation in rat carotid arteries after balloon injury. Circulation. 2001; 103: 133-139

10) Schnyder G, Roffi M, Flammer Y, Pin R, Hess OM: Association of plasma homocysteine with restenosis after percutaneous coronary angioplasty. Eur Heart J. 2002; 23: 726-733

11) Schnyder G, Flammer Y, Roffi M, Pin R, Hess OM: Plasma homocysteine levels and late outcome after coronary angioplasty. J Am Coll Cardiol. 2002; 40: 1769-1776

12) Zairis MN, Ambrose JA, Manousakis SJ, Stefanidis AS, 
Papadaki OA, Bilianou HI, DeVoe MC, Fakiolas CN, Pissimissis EG, Olympios CD, Foussas SG: The impact of plasma levels of C-reactive protein, lipoprotein (a) and homocysteine on the long-term prognosis after successful coronary stenting. J Am Coll Cardiol. 2002; 40: 13751382

13) Mccully KS: Vascular pathology of homocysteinemia: implications for the pathogenesis of arteriosclerosis. Am J Pathol. 1969; 56: 111-128

14) Arnesen E, Refsum H, Bønaa KH, Ueland PM, Førde $\mathrm{OH}$, Nordrehaug JE: Serum total homocysteine and coronary heart disease. Int J Epidemiol. 1995; 24: 704-709

15) Malinow MR, Nieto FJ, Szklo M, Chambless LE, Bond G: Carotid artery intimal-medial wall thickening and plasma homocyst(e)ine in asymptomatic adults. The atherosclerosis risk in communities study. Circulation. 1993; 87: 1107-1113

16) Ridker PM, Hennekens CH, Selhub J, Miletich JP, Malinow MR, Stampfer MJ: Interrelation of hyperhomocyst(e)inemia, factor $\mathrm{v}$ leiden, and risk of future venous thromboembolism. Circulation. 1997; 95: 1777-1782

17) Tawakol A, Omland T, Gerhard M, Wu JT, Creager MA: Hyperhomocyst(e)inemia is associated with impaired endothelium-dependent vasodilation in humans. Circulation. 1997; 95: 1119-1121

18) Majors A, Ehrhart LA, Pezacka EH: Homocysteine as a risk factor for vascular disease: Enhanced collagen production and accumulation by smooth muscle cells. Arterioscler Thromb Vasc Biol. 1997; 17: 2074-2081

19) Bellamy MF, McDowell IF: Putative mechanisms for vascular damage by homocysteine. J Inherit Metab Dis. 1997; 20: 307-315

20) M. Cheng, H. Ho, J. Lin, Y. Chen, E. Chan, and D. T. Chiu: Clinical relevance of plasma homocysteine levels in Taiwanese patients with coronary artery disease. Biofactors. 2008; 34: 125-134

21) O. Akyürek, E. Akbal, and F. Güneş: Increase in the Risk of ST Elevation Myocardial Infarction Is Associated With Homocysteine Level. Arch. Med. Res. 2014; 45: 501-506

22) O. Tang, J. Wu, and F. Qin: Relationship between methylenetetrahydrofolate reductase gene polymorphism and the coronary slow flow phenomenon. Coron. Artery Dis. 2014; 25: 653-657

23) H. Tanriverdi, H. Evrengul, Y. Enli, O. Kuru, D. Seleci, S. Tanriverdi, N. Tuzun, H. A. Kaftan, and N. Karabulut: Effect of homocysteine-induced oxidative stress on endothelial function in coronary slow-flow. Cardiology. 2007; 107: 313-320

24) C. L. Chao, H. H. Tsai, C. M. Lee, S. M. Hsu, J. T. Kao, K. L. Chien, F. C. Sung, and Y. T. Lee: The graded effect of hyperhomocysteinemia on the severity and extent of coronary atherosclerosis. Atherosclerosis. 1999; 147: 379-386

25) N. Hirose, Y. Arai, T. Ishii, M. Tushima, and J. Li: Association of mild hyperhomocysteinemia with aortic calcification in hypercholesterolemic patients. J. Atheroscler. Thromb. 2001; 8: 91-94

26) J. Li, S. Chai, C. Tang, and J. Du: Homocysteine potentiates calcification of cultured rat aortic smooth muscle cells. Life Sci. 2003; 74: 451-461

27) H. Xu, C. Liu, and Q. Wang: Plaque image characteristics, hyperhomocysteinemia, and gene polymorphism of homocysteine metabolism-related enzyme (MTHFR C677T) in acute coronary syndrome. Cell Biochem. Biophys.2013; 2: 403-407

28) J. L. Duan, T. Murohara, H. Ikeda, K. Sasaki, S. Shintani, T. Akita, T. Shimada, and T. Imaizumi: Hyperhomocysteinemia impairs angiogenesis in response to hindlimb ischemia. Arterioscler. Thromb. Vasc. Biol. 2000; 20: 25792585

29) Kosokabe T, Okumura K, Sone T, Kondo J, Tsuboi H, Mukawa H, Tomida T, Suzuki T, Kamiya H, Matsui H, Hayakawa T: Relation of a common methylenetetrahydrofolate reductase mutation and plasma homocysteine with intimal hyperplasia after coronary stenting. Circulation. 2001; 103: 2048-2054

30) Nygård O, Nordrehaug JE, Refsum H, Ueland PM, Farstad M, Vollset SE: Plasma homocysteine levels and mortality in patients with coronary artery disease. N Engl J Med. 1997; 337: 230-236

31) Stubbs PJ, Al-Obaidi MK, Conroy RM, Collinson PO, Graham IM, Noble IM: Effect of plasma homocysteine concentration on early and late events in patients with acute coronary syndromes. Circulation. 2000; 102: 605610

32) Lonn E, Yusuf S, Arnold MJ, Sheridan P, Pogue J, Micks M, McQueen MJ, Probstfield J, Fodor G, Held C, Genest J Jr; Heart Outcomes Prevention Evaluation (HOPE) 2 Investigators: Homocysteine lowering with folic acid and $B$ vitamins in vascular disease. N Engl J Med. 2006; 354: 1567-1578

33) Bønaa KH, Njølstad I, Ueland PM, Schirmer H, Tverdal A, Steigen T, Wang H, Nordrehaug JE, Arnesen E, Rasmussen K; NORVIT Trial Investigators: Homocysteine lowering and cardiovascular events after acute myocardial infarction. N Engl J Med. 2006; 354: 1578-1588

34) Toole JF, Malinow MR, Chambless LE, Spence JD, Pettigrew LC, Howard VJ, Sides EG, Wang CH, Stampfer M: Lowering homocysteine in patients with ischemic stroke to prevent recurrent stroke, myocardial infarction, and death: the Vitamin Intervention for Stroke Prevention (VISP) randomized controlled trial. JAMA. 2004; 291: $565-575$

35) Qin X, Huo Y, Xie D, Hou F, Xu X, Wang X: Homocysteine-lowering therapy with folic acid is effective in cardiovascular disease prevention in patients with kidney disease: a meta-analysis of randomized controlled trials. Clin Nutr. 2013; 32: 722-727

36) M. V. Holmes, P. Newcombe, J. A. Hubacek, R. Sofat, S. L. Ricketts, J. Cooper, M. M. Breteler, L. E. Bautista, P. Sharma, J. C. Whittaker, L. Smeeth, F. G. R. Fowkes, A. Algra, V. Shmeleva, Z. Szolnoki, M. Roest, M. Linnebank, J. Zacho, M. A. Nalls, A. B. Singleton, L. Ferrucci, J. Hardy, B. B. Worrall, S. S. Rich, M. Matarin, P. E. Norman, L. Flicker, O. P. Almeida, F. M. Van Bockxmeer, H. Shimokata, K. T. Khaw, N. J. Wareham, M. Bobak, J. A. C. Sterne, G. D. Smith, P. J. Talmud, C. Van Duijn, S. E. Humphries, J. F. Price, S. Ebrahim, D. A. Lawlor, G. J. Hankey, J. F. Meschia, M. S. Sandhu, A. D. Hingorani, and J. P. Casas: Effect modification by population dietary folate on the association between MTHFR genotype, homocysteine, and stroke risk: A meta-analysis of genetic studies and randomised trials. Lancet. 2011; 378: 584-594 